\title{
Histomorphometric Study of Implants Initially Stabilized through Bone GraftPacking into the Osteotomy before Implant Placement in Case of Wide Defects
}

\author{
Wang-Jae Lee', Ki-Seok Hong'
}

1. Department of Periodontology, Dankook University School of Dentistry, Cheonan, Korea

\section{Corresponding Author}

\section{Ki-Seok Hong, DDS, PhD}

Department of Periodontology, Dankook University School of Dentistry, San 29 Anseo-dong, Dongnamgu, Cheonan 330-714, Korea

TEL : +82-41-550-1987 FAX : +82-41-555-0222 E-mail : periohong@gmail.com

\section{Received for publication November 30, 2011; Returned after revision December 9, 2011;}

Accepted for publication December 16, 2011

\section{Acknowledgment}

The present research was conducted by the research fund of Dankook University in 2009.

\begin{abstract}
- Abstract
Purpose: This study sought to evaluate the effects of bone graft wedging on the initial stability of implants in bone sites of unfavorable quality.

Materials and Methods: Three male beagle dogs were used in this study. Osteotomies were performed with parallel drills $(\varnothing 4.1 \times 10 \mathrm{~mm})$, and fixtures $(\varnothing 3.3 \times 8 \mathrm{~mm})$ were placed. The control group was given implants without bone graft. Experiment group A was given implants with minimal initial stability using autobone grafts, whereas experiment group B was given xenografts. Groups were also divided by healing times at 4,8 , and 12 weeks.

Results: All implants in the control group failed to osseointegrate. On the other hand, all implants in the experiment groups were clinically well-maintained during the entire experiment period. After 4, 8, and 12 weeks, bone-to-implant contact (BIC) ratio and implant stability quotient (ISQ) increased in the experiment groups. The differences between experiment groups A and B were not statistically significant, however.

Conclusion: In unfavorable bone regions for dental implants, bone graft packing into the osteotomy prior to implant placement secured minimal initial stability and showed reasonable BIC ratios and ISQ values throughout the study period.
\end{abstract}

\section{- Key word : Dental implants, Bone substitutes}

\section{- J Kor Dent Sci. 2011; 4(2) : 67 - 72}

(c) This is an open access article distributed under the terms of the Creative Commons Attribution Non-Commercial License (http:// creativecommons.org/licenses/by-nc/3.0) which permits unrestricted non-commercial use, distribution, and reproduction in any medium, provided the original work is properly cited. 


\section{Introduction}

There is general consensus that there are certain influential factors that affect the success of dental implant treatments, such as the timing of the implant therapy. In particular, the timing of implant replacement (i.e., either delayed or immediate) depends on various clinical situations particularly the quality and quantity of bone available at the implant site ${ }^{1-3)}$. For successful dental implant therapy, initial stability of the implant is usually required ${ }^{4-8)}$. Note, however, that it is often difficult to anchor the implant in poorquality bone. To overcome this problem, many surgeons have suggested the use of self-tapping implants - which does away with surgical tapping in a prepared osteotomy -to achieve higher implant survival rate ${ }^{9)}$.

Another approach to enhancing the initial stability of implants in poor-quality bones is to place a tapered implant into a standard, parallel-sided osteotomy. The principle behind this approach is to induce controlled compressive forces in the cortical bone layer as the implant is inserted; these forces will increase the initial stability of the implant and transfer the region of the highest stress/strain to the cortical layer, where it will be better tolerated ${ }^{10,11)}$.

Some surgical techniques were introduced to improve bone density and quality of the implant site for initial stability $^{12-14}$. One of these techniques is the osteotome technique, which was designed to increase the initial stability of dental implants in the maxillary molar area ${ }^{15)}$.

In some cases, despite these various attempts, it is not easy to improve the initial stability of implants in poor-quality bones with limited available lengths such as the inferior alveolar canal. Therefore, this study sought to evaluate the effects of bone graft packing into the osteotomy prior to implant placement on the initial stability of implants in sites with unfavorable bone quality and limited available bone length.

\section{Materials and Methods}

\section{Surgical Procedures}

Three male beagle dogs weighing approximately $35 \mathrm{~kg}$ were used in this study. The animals had intact mandibles, no oral viral or fungal lesions, and good general health. Animal selection and management, surgical protocol, and preparation were carried out according to the guidelines approved by the Institutional Animal Care and Use Com- mittee of Dankook University, Cheonan, Korea.

In the first stage of the study, the dogs were anesthetized under sterile conditions using $2 \mathrm{mg} / \mathrm{kg}$ of xylazine (Rompun $^{\circledR}$, Bayer Korea, Seoul, Korea) and $10 \mathrm{mg} / \mathrm{kg}$ IV ketamine hydrochloride (Ketalar ${ }^{\circledR}$, Yuhan Co., Seoul, Korea). A full-thickness flap was reflected, and all premolars (P1 P4) were carefully extracted. The flap was subsequently sutured with 5-0 resorbable suture material (Polyglactin 910 braided absorbable suture, Ethicon, Johnson \& Johnson Int., Edinburgh, UK) using the interrupted suturing technique. After the surgery, the subjects received $10 \mathrm{mg} / \mathrm{kg}$ IV of the antibiotic amikacin sulfate (Amiktam inj ${ }^{\circledR}$, Kunwha Pharm., Seoul, Korea).

After the 6-week healing period, the $\varnothing 3.3 \times 8 \mathrm{~mm}$ fixtures (TIS, Seoul, Korea) were placed into the extracted sites under the same surgical conditions described previously. Mid-crestal incision was made to promote primary closure, with the mucoperiosteal flap carefully reflected. The surgical site was carefully flattened with a small round diamond burr and irrigated with sterile saline. Implant osteotomies (diameter: $4.1 \mathrm{~mm}$, depth: $10 \mathrm{~mm}$ ) were created using a surgical parallel twist drill at 1,000 rpm with chilled saline irrigation according to the manufacturer's instructions. The same procedure was performed on the other side

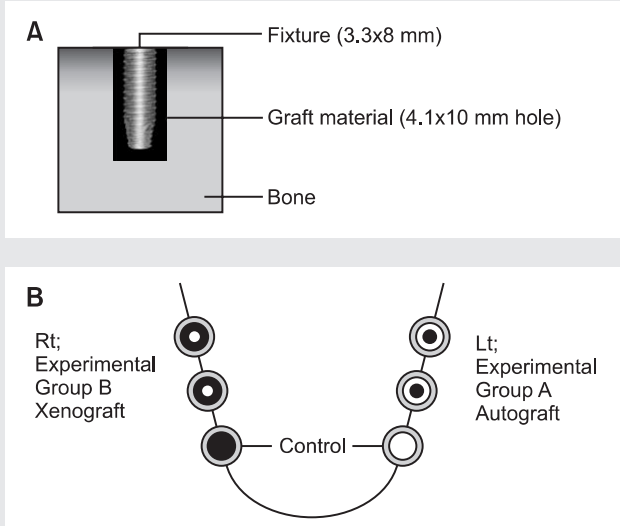

Figure 1. Schematic drawing of the experiment design. (A) Implant osteotomies ( $\varnothing 4.1 \mathrm{~mm}$, depth of $10 \mathrm{~mm}$ ) were created, and $\varnothing 3.3 \times 8 \mathrm{~mm}$ fixtures were placed using the bone graft wedging technique. (B) Experiment group A was given autobone graft implants with minimal initial stability, whereas experiment group B was given xenograft implants (OCS-B ${ }^{\circledR}$ ).

Wang-Jae Lee, et al: Histomorphometric Study of Implants Initially Stabilized through Bone Graft Packing into the Osteotomy before Implant Placement in Case of Wide Defects. J Kor Dent Sci 2011. 
of the mandible. The control group was given implants without bone graft. To secure initial stability, bone graft materials were packed into the osteotomy prior to implant placement in the experiment groups. Autograft materials were used in experiment group A, and xenograft (OCS-B ${ }^{\circledR}$, Nibec, Seoul, Korea) materials, in experiment group B (Fig. 1A). The fixtures were inserted either with hand wrenching or by using a surgical hand piece (Fig. 1B). After fixture installation, cover screws were carefully connected to the fixtures. Releasing incision was made for the primary closure, and flaps were closed with 5 0 resorbable suture materials. The same post-operative care for tooth extraction was administered. Sutures were removed after 7 10 days, and soft diet was provided throughout the study period.

\section{Sample Retrieval}

Subjects were sacrificed at 4, 8, and 12 weeks after the second surgery, and implant stability quotient (ISQ) measurements for the fixtures were performed using an Osstell ${ }^{\mathrm{TM}}$ Mentor device (Integration Diagnostics AB, Göteborg, Sweden) at each time point. Euthanasia was performed through anesthesia drug overdose. The implants and the surrounding bone were harvested en bloc and fixed in $10 \%$ neutral buffered formalin. The specimens were dehydrated in $70 \%, 90 \%, 95 \%$, and $100 \%$ alcohol, embedded in methacrylate, and sectioned along the long axis of the implants in the midline using a diamond saw (Mode1650 ${ }^{\circledR}$, South Bay Technology, San Clemente, CA, USA).

From each implant site, the central section was reduced to a final thickness of approximately $50 \mu \mathrm{m}$ by microgrinding and polishing before being stained with toluidine blue. The images were assessed at magnification of $100 \times$ and digitized. Image Processing Tool Kit (IPTK) version 5.0 (Reindeer Graphics Co., Asheville, NC, USA) was used for the

\begin{tabular}{lcc}
\multicolumn{3}{c}{ Table 1. Bone-to-implant contact ratios (mean \pm SD in \%) } \\
\hline & Group A & Group B \\
\hline 4 weeks & $39.6 \pm 6.4^{\text {a }}$ & $36.8 \pm 7.6^{\text {b }}$ \\
8 weeks & $48.3 \pm 5.4$ & $44.6 \pm 6.2$ \\
12 weeks & $51.7 \pm 7.3$ & $50.2 \pm 7.9$ \\
Average & $46.5 \pm 3.4$ & $43.8 \pm 8.7$ \\
\hline
\end{tabular}

aStatistically significant difference from the ratios at 8 and 12 weeks $(P<0.05)$. bStatistically significant difference from the ratios at 8 and 12 weeks $(P<0.05)$.

Wang-Jae Lee, et al: Histomorphometric Study of Implants Initially Stabilized through Bone Graft Packing into the Osteotomy before Implant Placement in Case of Wide Defects. J Kor Dent Sci 2011. image analysis. Bone-implant contact (BIC) ratio was defined as the percentage of direct BIC with respect to the overall implant surface.

\section{Statistical Analysis}

To compare the differences in ISQ values and BIC ratios between the graft materials at each healing time point, Mann-Whitney U test was performed. Next, to compare the differences in ISQ values and BIC ratios among healing times in each group, Wilcoxon rank-sum test was performed. A P-value of 0.05 was set for statistical significance.

\section{Results}

\section{Clinical Examination}

All implants in the control group failed to osseointegrate within 4 weeks. On the other hand, all implants in the experiment groups were clinically well maintained during the entire experiment period.

\section{Histomorphometric Analysis}

The results of the histomorphometric analysis are presented in Table 1. The average BIC ratio was $46.5 \pm 3.44 \%$ in experiment group A and $43.8 \pm 6.49 \%$ in experiment group B; although the BIC ratios of experiment group A were greater than those of experiment group B, the difference was not statistically significant.
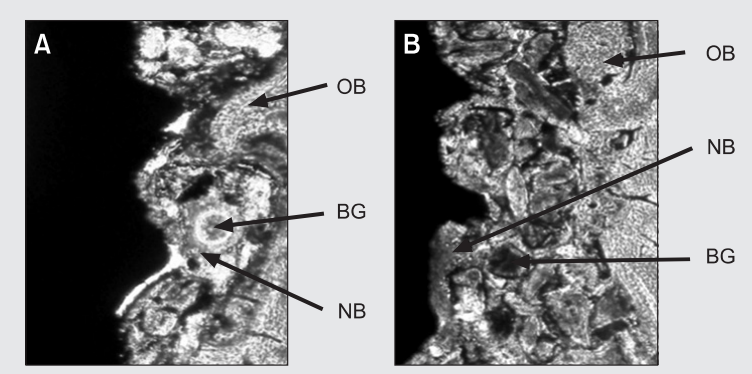

Figure 2. Histological presentation of the implant site after 4 weeks' healing. In the groups, a limited amount of new bone can be seen (toluidine blue stain, 40x magnification). A: Experiment group A, B: Experiment group B, NB: new bone, BG: bone graft, $\mathrm{OB}$ : old bone

Wang-Jae Lee, et al: Histomorphometric Study of Implants Initially Stabilized through Bone Graft Packing into the Osteotomy before Implant Placement in Case of Wide Defects. J Kor Dent Sci 2011. 

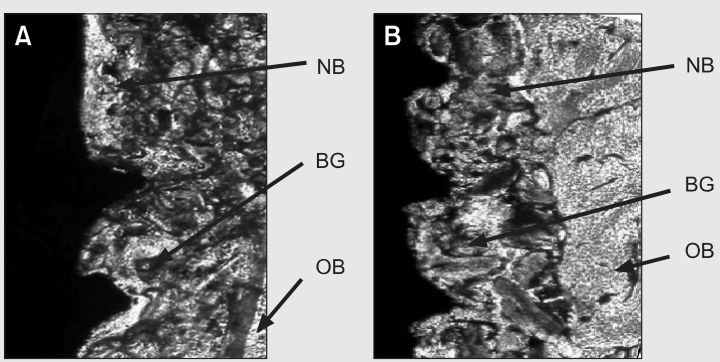

Figure 3. Histological presentation of the implant site after 8 weeks' healing. In the groups, newly formed bone in the interthread space was in contact with the implant (toluidine blue stain, 40x magnification). A: Experiment group A, B: Experiment group B, NB: new bone, BG: bone graft, OB: old bone.

Wang-Jae Lee, et al: Histomorphometric Study of Implants Initially Stabilized through Bone Graft Packing into the Osteotomy before Implant Placement in Case of Wide Defects. J Kor Dent Sci 2011
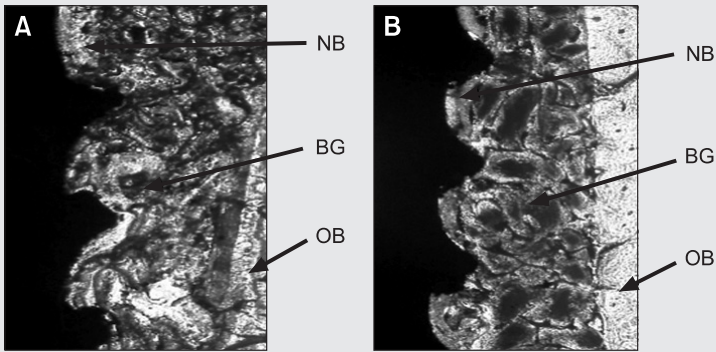

Figure 4. Histological presentation of the implant site after 12 weeks' healing. In the groups, broad-based contacts could be observed from the tops of the threads to the valleys of the threads (toluidine blue stain, 40x magnification). A: Experiment group A, B: Experiment group B, NB: new bone, BG: bone graft, OB: old bone.

Wang-Jae Lee, et al: Histomorphometric Study of Implants Initially Stabilized through Bone Graft Packing into the Osteotomy before Implant Placement in Case of Wide Defects. J Kor Dent Sci 2011.

The relative improvement of the BIC ratio over time was similar between both experiment groups (Fig. 2 4). Between 4 weeks and 8 and 12 weeks, the BIC ratio increased in both experiment groups, and the difference was statistically significant $(\mathrm{P}<0.05)$. Note, however, that there was no statistically significant difference in the BIC ratios between 8 and 12 weeks. The differences in the BIC ratios between experiment groups A and B at 4, 8, and 12 weeks were not statistically significant.
Table 2. ISQ values measured at various time points throughout the healing period in each group (mean $\pm S D$ )

\begin{tabular}{ccc}
\hline & Group A & Group B \\
\hline 4 weeks & $60 \pm 4.7$ & $57 \pm 5.8$ \\
8 weeks & $67 \pm 6.3$ & $64 \pm 3.8$ \\
12 weeks & $69 \pm 4.5$ & $66 \pm 4.8$ \\
Average & $65 \pm 4.3$ & $62 \pm 3.7$ \\
\hline
\end{tabular}

ISQ: implant stability quotient. No significant differences were found between the groups at any healing time points.

Wang-Jae Lee, et al: Histomorphometric Study of Implants Initially Stabilized through Bone Graft Packing into the Osteotomy before Implant Placement in Case of Wide Defects. J Kor Dent Sci 2011

\section{Resonance Frequency Analysis}

The results of the resonance frequency analysis are presented in Table 2. No data were obtained at week 0 . At weeks 4,8 , and 12 of the healing period, no statistically significant differences were found in the ISQ value within the experiment groups, and the results were similar between the experiment groups at each time point. From week 4 to weeks 8 and 12, an increase in the ISQ value was found in both experiment groups, but the difference was not statistically significant.

\section{Discussion}

Initial implant stability is an important factor in successful osseointegration, since implant instability results in fibrous encapsulation and failure of the implant ${ }^{16)}$. Furthermore, longer healing period is necessary for implants placed in low-density bone ${ }^{17,18)}$.

In this study, BIC ratios and ISQ values at weeks 8 and 12 of the healing period increased in comparison to week 4 of the healing period. Note, however, that there were no differences in the BIC ratios and ISQ values at each healing period between experiment groups A and B. These results suggest that the healing time is more important than the type of graft material used for successful osseointegration. This observation confirms the hypothesis, i.e., longer healing period is necessary for implants placed in lowdensity bone.

Recently, many clinicians have taken an interest in immediate implant therapy because the total treatment time may be shorter and residual socket walls may be preserved. ${ }^{19-24}$ Note, however, that the required initial stability for this therapy is usually not secured due to poor bone quality; therefore, surgeons have to wait months before placing implants 
in regions with poor bone quality. In 1994, Summers ${ }^{15}$ ) introduced the osteotome technique to overcome the challenges associated with unfavorable implant sites. The objective of this technique is to preserve the entire existing bone by minimizing or even eliminating the drilling sequence. The osteotomy site is progressively compacted with osteotome instruments of various sizes. The resulting improved bone density helps increase initial implant stability in low-density bone. This technique seems promising for low-density bone, but surgeons often fail to secure initial stability in sites with limited available bone length and unfavorable bone quality. In these circumstances, bone graft packing into unfavorable bone for implant placement can be considered, possibly even shortening the total treatment time.

The size of the gap between the bony wall and the implant is another important factor for successful osseointegration. Several studies have reported the relationship between gap width and healing around immediate implants. Knox et al. ${ }^{25}$ proved that gaps larger than $1 \mathrm{~mm}$ resulted in less direct BIC. Carlsson et al. ${ }^{26)}$ showed that there was no osseointegration seen histologically when the gap between the bone and the implant was larger than $0.35 \mathrm{~mm}$. Wilson et al. ${ }^{27)}$ demonstrated that there was no need to use a membrane for a gap less than $0.5 \mathrm{~mm}$, whereas no integration between the bone and the implant was observed if the gap width exceeded $4 \mathrm{~mm}$. These studies were performed after the initial stability of the fixture was established. In this study, however, graft materials were packed into the gap to achieve initial stability. Similar to the results of other studies investigating gap sizes smaller than $0.5 \mathrm{~mm}$, all groups in this study showed successful osseointegration both clinically and histologically. Nonetheless, further studies on the long-term effects of this technique or its use in cases of larger gap sizes between the bone and the implant are needed.

Botticelli et al. ${ }^{28-32)}$ suggested that the implant surface could affect the healing pattern of gap defects around implants. The SLA rough surface implant was used in their study, showing better healing pattern than implants with smooth surfaces. Therefore, in this study, an implant with rough surface (RBM) was chosen instead of the implant with smooth surface. Consequently, successful osseointegration was observed in both experiment groups. Further studies regarding the implant surface used in this protocol are needed, however.

There are several recommended protocols for implant placement in low-density bone. The most important point is the precise surgical preparation of the implant sites; the implant direction should be unchanged during drilling. Additionally, drilling over the entire implant length is not recommended. During implant installation, light force should be used, and rough implant surfaces are recommended to improve bone healing. In this study, implant osteotomies were made wider than the planned fixture diameters. The initial stability of the implant was secured by packing graft materials prior to implant placement into the osteotomy. In experiment group B, xenograft material, which was grittier and harder, was used; obtaining implant stability was difficult. In experiment group A, autobone graft materials -- which were softer -were used.

For this surgical protocol, the use of soft graft materials allowed for plasticity by pressure. There was not enough initial stability in either group, however, to connect the equipment to measure ISQ values. Therefore, we measured ISQ values only at weeks 4,8 , and 12 of the healing period in this study.

Bone graft packing into the osteotomy prior to implant placement in this study demonstrated reasonable bone-toimplant contact ratio and ISQ value throughout the experiment period. Longitudinal clinical studies are needed to confirm the effectiveness of this technique, however.

\section{Conclusion}

Bone graft packing into the osteotomy prior to implant placement in this study demonstrated reasonable BIC ratio and ISQ value throughout the experiment period. 
1. Jaffin RA, Berman CL. The excessive loss of Branemark fixtures in type IV bone: a 5-year analysis. J Periodontol. 1991; 62: 2-4.

2. Lekholm U, van Steenberghe D, Herrmann I, Bolender C, Folmer T, Gunne J, Henry PJ. Osseointegrated implants in the treatment of partially edentulous jaws: a prospective 5-year multicenter study. Int J Oral Maxillofac Implants. 1994; 9: 627-35.

3. Friberg B, Nilson H, Olsson M, Palmquist C. Mk II: the self-tapping Brånemark implant: 5-year results of a prospective 3-center study. Clin Oral Implants Res. 1997; 8: 279-85.

4. Albrektsson T, Brånemark PI, Hansson HA, Lindström J. Osseointegrated titanium implants. Requirements for ensuring a long-lasting, direct bone-to-implant anchorage in man. Acta Orthop Scand. 1981; 52: $155-70$

5. Orenstein IH, Tarnow DP, Morris HF, Ochi S. Factors affecting implant mobility at placement and integration of mobile implants at uncovering. J Periodontol. 1998; 69: 1404-12.

6. Jemt T. Failures and complications in 391 consecutively inserted fixed prostheses supported by Brånemark implants in edentulous jaws: a study of treatment from the time of prosthesis placement to the first annual checkup. Int J Oral Maxillofac Implants. 1991; 6: 270-6.

7. Truhlar RS, Orenstein IH, Morris HF, Ochi S. Distribution of bone quality in patients receiving endosseous dental implants. J Oral Maxillofac Surg. 1997; 55(12 Suppl 5): 38-45.

8. Friberg B, Jemt T, Lekholm U. Early failures in 4,641 consecutively placed Brånemark dental implants: a study from stage 1 surgery to the connection of completed prostheses. Int J Oral Maxillofac Implants. 1991; 6: 142-6.

9. Ivanoff CJ, Gröndahl K, Bergström C, Lekholm U, Brånemark PI. Influence of bicortical or monocortical anchorage on maxillary implant stability: a 15-year retrospective study of Brånemark System implants. Int J Oral Maxillofac Implants. 2000; 15: 103-10.

10. Martinez H, Davarpanah M, Missika P, Celletti R, Lazzara R. Optima implant stabilization in low density bone. Clin Oral Implants Res. 2001; 12: 423-32.

11. O'Sullivan D, Sennerby L, Meredith N. Influence of implant taper on the initial and secondary stability of osseointegrated titanium implants. Clin Oral Implants Res. 2004; 15: 474-80.

12. Lundgren D, Lundgren AK, Sennerby L. The effect of mechanical intervention on jaw bone density. Clin Oral Implants Res. 1995; 6: $54-9$

13. Matsumoto H, Ochi M, Abiko Y, Hirose Y, Kaku T, Sakaguchi K. Pulsed electromagnetic fields promote bone formation around dental implants inserted into the femur of rabbits. Clin Oral Implants Res. 2000; 11: 354-60

14. Nkenke E, Kloss F, Wiltfang J, Schultze-Mosgau S, Radespiel-Tröger M, Loos K, Neukam FW. Histomorphometric and fluorescence microscopic analysis of bone remodelling after installation of implants using an osteotome technique. Clin Oral Implants Res. 2002; 13: 595 602 .

15. Summers RB. A new concept in maxillary implant surgery: the osteotome technique. Compendium. 1994; 15: 152, 154-6, 158.

16. Lioubavina-Hack N, Lang NP, Karring T. Significance of primary stability for osseointegration of dental implants. Clin Oral Implants Res. 2006; 17: 244-50

17. Friberg B, Sennerby L, Linden B, Gröndahl K, Lekholm U. Stability measurements of one-stage Brånemark implants during healing in mandibles. A clinical resonance frequency analysis study. Int J Oral Maxillofac Surg. 1999; 28: 266-72.

18. Johansson CB, Albrektsson T. A removal torque and histomorphometric study of commercially pure niobium and titanium implants in rabbit bone. Clin Oral Implants Res. 1991; 2: 24-9.

19. Watzek G, Haider R, Mensdorff-Pouilly N, Haas R. Immediate and delayed implantation for complete restoration of the jaw following extraction of all residual teeth: a retrospective study comparing different types of serial immediate implantation. Int J Oral Maxillofac Implants. 1995; 10: 561-7.

20. Denissen HW, Kalk W, Veldhuis HA, van Waas MA. Anatomic consideration for preventive implantation. Int J Oral Maxillofac Implants. 1993; 8: 191-6.

21. Lazzara RJ. Immediate implant placement into extraction sites: surgical and restorative advantages. Int J Periodontics Restorative Dent. 1989; 9: 332-43.

22. Werbitt MJ, Goldberg PV. The immediate implant: bone preservation and bone regeneration. Int J Periodontics Restorative Dent. 1992; 12 206-17.

23. Gelb DA. Immediate implant surgery: three-year retrospective evaluation of 50 consecutive cases. Int J Oral Maxillofac Implants. 1993; 8: 388-99.

24. Lang NP, Brägger U, Hämmerle CH, Sutter F. Immediate transmucosal implants using the principle of guided tissue regeneration. I. Rationale, clinical procedures and 30-month results. Clin Oral Implants Res. 1994; 5: 154-63.

25. Knox R, Caudill R, Meffert R. Histologic evaluation of dental endosseous implants placed in surgically created extraction defects. In J Periodontics Restorative Dent. 1991; 11: 364-75.

26. Carlsson L, Röstlund T, Albrektsson B, Albrektsson T. Implant fixation improved by close fit. Cylindrical implant-bone interface studied in rabbits. Acta Orthop Scand. 1988; 59: 272-5.

27. Wilson TG Jr, Schenk R, Buser D, Cochran D. Implants placed in immediate extraction sites: a report of histologic and histometric analyses of human biopsies. Int J Oral Maxillofac Implants. 1998; 13 333-41.

28. Botticelli D, Berglundh T, Lindhe J. Hard-tissue alterations following immediate implant placement in extraction sites. J Clin Periodontol. 2004; $31: 820-8$.

29. Botticelli D, Berglundh T, Buser D, Lindhe J. Appositional bone formation in marginal defects at implants. Clin Oral Implants Res. 2003; 14: 1-9.

30. Botticelli D, Berglundh T, Persson LG, Lindhe J. Bone regeneration at implants with turned or rough surfaces in self-contained defects. An experimental study in the dog. J Clin Periodontol. 2005; 32: 448-55.

31. Botticelli D, Berglundh T, Lindhe J. Resolution of bone defects of varying dimension and configuration in the marginal portion of the peri-implant bone. An experimental study in the dog. J Clin Periodontol. 2004; 31: 309-17.

32. Botticelli D, Berglundh T, Lindhe J. The influence of a biomaterial on the closure of a marginal hard tissue defect adjacent to implants. An experimental study in the dog. Clin Oral Implants Res. 2004; 15: 285 92. 